\title{
Developing Laser Ablation in an Electron Cyclotron Resonance Ion Source for Actinide Detection with AMS
}

\author{
W. Bauder ${ }^{1,2}$, R.C. Pardo ${ }^{1}$, F.G. Kondev ${ }^{1}$, S. Kondrashev ${ }^{1}$, C. Nair ${ }^{1}$, O. Nusair ${ }^{1}$, T. Palchan ${ }^{1}$, R.

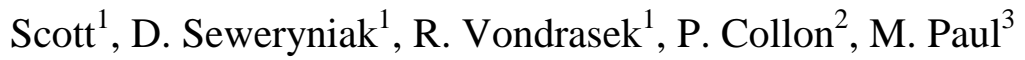 \\ ${ }^{1}$ Argonne National Laboratory, Physics Division, 9600 S. Cass Ave, Lemont, IL 60439, USA \\ ${ }^{2}$ University of Notre Dame, Nuclear Science Laboratory, 124 Nieuwland Science Hall, Notre \\ Dame, IN 46556, USA \\ ${ }^{3}$ Hebrew University, Racah Institute of Physics, Jerusalem 91904, Israel
}

\begin{abstract}
A laser ablation material injection system has been developed at the ATLAS electron cyclotron resonance (ECR) ion source for use in accelerator mass spectrometry experiments. Beam production with laser ablation initially suffered from instabilities due to fluctuations in laser energy and cratering on the sample surface by the laser. However, these instabilities were rectified by applying feedback correction for the laser energy and rastering the laser across the sample surface. An initial experiment successfully produced and accelerated low intensity actinide beams with up to 1000 counts per second. With continued development, laser ablation shows promise as an alternative material injection scheme for ECR ion sources and may help substantially reduce cross talk in the source.
\end{abstract}

keywords: Accelerator Mass Spectrometry; Electron Cyclotron Resonance Ion Source; Actinides 


\section{Introduction}

The assessment of Generation IV reactors and advanced fuel cycles requires extensive knowledge of transuranic neutron capture cross sections [1]. Presently, data with smaller uncertainties are needed for a variety of actinide isotopes (Plutonium, Americium, Curium, up to Californium) in order to optimize elements of the nuclear fuel cycle for feasibility studies (e.g. neutron doses at fuel fabrication, decay heat in a repository, etc) [2]. However, the current data for minor actinides, especially in the fast-neutron region, are sparse and, in some cases, based only on models. The Measurement of Actinide Neutron TRAnsmutations (MANTRA) project aims to improve these data by measuring energy-integrated neutron capture cross sections across the actinide region. The project, a collaboration between Idaho National Laboratory (INL) and Argonne National Laboratory (ANL), will extract neutron capture cross sections by:

1. Irradiating isotopically pure actinide samples in the Advanced Test Reactor (ATR) at INL. Samples of interest for ATLAS are: ${ }^{232} \mathrm{Th},{ }^{236} \mathrm{U},{ }^{237} \mathrm{~Np},{ }^{238} \mathrm{U},{ }^{242} \mathrm{Pu},{ }^{243} \mathrm{Am},{ }^{244} \mathrm{Pu}$, ${ }^{248} \mathrm{Cm}$

2. Measuring isotopic concentrations due to successive neutron captures on actinide materials using Accelerator Mass Spectrometry (AMS) at the Argonne Tandem Linear Accelerator System (ATLAS)

3. Using measured isotopic ratios to extract integral cross sections.

For an isotopically pure sample of mass A, the neutron capture cross section can, to a good approximation, be written as [3]

$$
\sigma_{A}^{c}=\frac{\left(\frac{N_{A+1}(t)}{N_{A}(t)}\right)-\left(\frac{N_{A+1}(0)}{N_{A}(0)}\right)}{\tau}
$$


Where $\left(\frac{N_{A+1}(t)}{N_{A}(t)}\right)$ is the concentration of nuclei with mass $\mathrm{A}+1$ after irradiating for a time $\mathrm{t}$, $\left(\frac{N_{A+1}(0)}{N_{A}(0)}\right)$ is the concentration before irradiation, and $\tau$ is the neutron fluence. Similar formulae can be derived for two and three neutron capture cross sections, although the isotopic ratios get progressively smaller. The superior sensitivity of AMS, however, allows us to measure multiple neutron capture cross sections from a single sample with only a relatively short irradiation time (50-100 days in the ATR). Thus, we can, in principle, infer multiple cross sections from a single sample, and from a relatively small selection of samples, we can make a comprehensive set of cross section measurements. Additionally, MANTRA will undertake three different irradiations using different neutron filters which cut out various portions of the thermal neutron spectrum. Thus, the actinides will be irradiated in an epithermal and a pure fast neutron spectra. A combination of measurements on the same base material with different neutron filters can provide some information about the structure of the differential cross section, $\sigma(\mathrm{E})$, in the fast neutron regime. The key to measuring all these cross sections with limited irradiation time and a limited number of initial actinide samples is to analyze the reaction products with AMS.

Accelerator Mass Spectrometry is a widely expanding field of ultrasensitive mass spectrometry that has found uses across the physical, biological and earth sciences [4,5]. AMS looks primarily at long-lived radioactive isotopes that are inaccessible through traditional techniques like gamma spectroscopy. For many isotopes, sensitivities have stretched down to 1 part in $10^{15}$ with as few as $10^{4}$ atoms of interest in a sample [6]. This offers higher sensitivity than conventional mass spectrometry, and extremely high discrimination power from natural backgrounds.

The use of AMS for isotopic analysis in MANTRA is a key innovation because its sensitivity allows for more cross section measurements from less sample material. Smaller 


\section{AMS at ATLAS}

Figure 1 shows a floor plan of ATLAS with the components relevant to MANTRA highlighted. Multiply-charged, positive ions are produced in the electron cyclotron resonance (ECR) ion source. The ions are accelerated through the first section of accelerating elements in the Positive Ion Injector (PII), to $1 \mathrm{MeV} / \mathrm{u}$ and then detected using the Fragment Mass Analyzer (FMA). The FMA, with a unique capability of analyzing fast ions by mass-to-charge (m/q) ratio with ultra-high sensitivity [7,8] constitutes a key component of the ATLAS AMS setup for this experiment and justifies, in our opinion, the use of a complex facility such as ATLAS. Although greater beam energy would help with detection, especially trying to separate potential isobar contaminants, the rigidity acceptance of the FMA limits the beam energy. In order to measure the isotopic ratio, we must measure both the original actinide material, $\mathrm{A}$, and the neutron capture produced actinide, $\mathrm{A}+1, \mathrm{~A}+2$, etc.

Past AMS experiment at ALTAS typically looked at only a handful of samples (typically 1-5 samples), and measured only one isotopic ratio for all the samples [7,8]. For MANTRA, we need to look at over 30 samples, and each one requires 2-3 different isotopic ratio measurements. This requires continual and consistent scalings of the accelerator between different masses while maintaining a measured, stable accelerator transmission between the scalings. Additionally, the multitude of samples with overlapping mass regions increases the risk of cross talk in the source. For example, residual material in the source from a ${ }^{238} \mathrm{U}$ sample will interfere with the 
measurement of the $\mathrm{A}+1$ region in a ${ }^{237} \mathrm{~Np}$ sample. Thus, in order to contain cross-talk and maintain machine stability while scaling between masses and samples, we have made several modifications to the AMS setup at ATLAS:

1. Creating a centralized "clock" program to control automated scaling of the accelerator between different isotope settings.

2. Adding a multi-sample changer to the ion source to rapidly switch between up to 20 samples without breaking vacuum.

3. Developing a laser ablation material injection setup for the ion source to help reduce cross-talk.

\section{Laser Ablation at the ECR}

Laser ablation has been developed as a technique for feeding material into an ICP-MS [9]. At Argonne laser ablation was first tested at the ECR in the early 1990's as an alternative to evaporating sample materials with ovens [10]. However, the use of ovens at ATLAS was eventually largely supplanted by a sputtering technique in which a solid sample was negatively biased to attract positive ions from the plasma to sputter material. This technique proved to be more efficient than using an oven, but also produced significant contamination. In particular, sputtering left a considerable amount of material on the ECR walls around the sample which is easily recycled by the plasma. Thus, for MANTRA, sputtering could produce considerable crosstalk between samples.

In order to reduce the cross-talk, we instead inject material with laser ablation. Figure 2 shows a cutaway of the ECR ion source and the ablation geometry [11]. The sputtering setup uses a sample inserted radially into the plasma chamber. This configuration seems to maximize 
sputtering production, but creates significant contamination. Our laser ablation setup places the sample slightly off the plasma axis at the rear of the plasma chamber, and the laser is focused through the source extraction hole and onto the sample surface. Initially, the sample was placed directly on-axis, but this severely reduced the performance of the source because it interfered with the plasma loss lines. To correct this problem, we moved the sample slightly off-axis and aligned the laser to ablate from this position.

The principle behind ablation involves super-heating a sample with an incoming laser pulse which instantaneously vaporizes the material. In offline testing, we showed that the rate of material ablation directly corresponds to the laser energy per pulse and the repetition rate of the laser [12]. Additionally, unlike sputtering, the ejected material comes off in a somewhat confined plume. Therefore, positioning the sample axially allows the plume to be ejected along the length of the chamber and, hopefully, be better consumed by the plasma.

Previous work with laser ablation has shown that if the laser pulse length is below the nanosecond regime, the ablation is in a non-equilibrium state and does not produce heating in the surrounding sample [13]. Additionally, when the sample lattice is heated by longer pulse length ablation, the ejected material composition consists of larger micron-sized particles which are not as easily consumed by the plasma [14]. So, in order to reduce sample heating during ablation, we selected a picosecond laser pulse length. Table I shows the details of the laser.

After initial offline testing, the laser was moved to the ECR2 ion source in early 2012. Figure 3 shows the floor plan of the laser and accompanying optics at the ECR source. Due to space constraints, the laser is mounted off the high voltage platform. To reach the sample in the ECR chamber, the laser enters through a window in the exit beamline of the ECR source and passes through the source extraction hole. A beam expander on the optical table produces a 
collimated beam which is then focused through a 4 meter focal length lens onto the sample surface. A CCD camera, aligned on a track parallel to the laser, images the sample in order to align the laser. Figure 3 shows a CCD image of the sample in the ECR. A backlight illuminates the sample edges for alignment purposes. When the laser is on target light from the laser induced plasma can be detected on the CCD to confirm the laser alignment.

\section{Laser Performance with ECR}

Following the laser installation at the ECR source, we began testing the interaction of laser ablation with the plasma. The source operates by ionizing neutral material through electron collisions between the plasma electrons and the atoms of interest. Our aim is simply to inject the ablated material into the plasma region to produce ions. However, the eventual beam production will depend upon the efficiency of the laser-ECR interaction. To effectively measure the MANTRA samples, we need to produce a beam of a few enA of actinide beam and, more importantly, maintain beam stability. Since the beam current is not monitored in real time, beam stability is important to minimize uncertainties while making measurements and switching between isotopes.

Two initial problems appeared during our initial testing. First, the laser showed energy instabilities over time, and because the ablation rate depends on the laser energy, the beam current showed corresponding oscillations. Second, even for periods of stable laser energy, the beam current output showed a characteristic falloff after only 20 minutes of ablation. Figure 4 shows the ion source output of $\mathrm{a}^{48} \mathrm{Ti}^{10+}$ beam compared with the laser energy over the same period. The beam current starts at nearly $5 \mathrm{e} \mu \mathrm{A}$ but quickly falls off to a few hundred enA where it is relatively stable. This effect has also been observed in LA-ICP-MS measurements and is 
attributed to the laser creating craters in the sample surface [15]. As the laser ablates material away, it creates a very well defined crater. As the crater becomes deeper, the ejected material becomes caught on the walls and cannot enter the plasma. At this point, the ablation process becomes increasingly inefficient. The combination of the sample cratering and laser instabilities makes it impossible to obtain the necessary beam production and stability.

In order to correct the laser instabilities we use a partially transparent mirror and a photodiode to measure the laser energy in real time. This signal can be used to create a feedback signal to correct the laser energy. We use the built-in attenuation adjustment in the laser head to automatically maintain a stable laser energy. Figure 5 shows a plot of the laser energy with and without feedback correction. The feedback maintains the laser energy to $\pm 2 \%$ at $1 \mathrm{~mJ}$. This correction has proven sufficient to avoid noticeable fluctuations in beam current.

With a stable laser energy output we can begin to address the cratering effect. In order to prevent the laser from creating a crater in one spot of the sample, we use a pair of piezoelectric actuators attached to a mirror to move the laser spot systematically across the sample surface. For simplicity, the laser moves in a $\sim 2 \mathrm{~mm}^{2}$ rectangular raster pattern, completing a full cycle across the sample surface every 100ms. Figure 6 shows an image of two samples; one without rastering the laser and one ablated with rastering. The rastering ablates material evenly from the sample surface eliminating the ablation craters seen on the titanium sample.

In terms of beam production, Figure 7 shows a comparison of beam production with and without rastering for an $\mathrm{Al}$ beam. Although the initial production is greater for a non-rastered sample, the rastering allows us to maintain a much more stable beam current, and the integrated beam production is generally greater, for long ablation times, than without rastering. With the 
addition of feedback for the laser energy and the rastering across the sample surface, we can produce a more stable beam with laser ablation.

\section{Accelerated Beam Measurements}

A first experiment with laser ablation beams was performed in March of 2013. The experiment was our first work with accelerated ablated beams, and it aimed to look at unirradiated MANTRA samples. Four samples were measured: ${ }^{238} \mathrm{U},{ }^{232} \mathrm{Th},{ }^{237} \mathrm{~Np}$, and ${ }^{248} \mathrm{Cm}$. We established the accelerator tune and measured the transmission with a ${ }^{84} \mathrm{Kr}^{13+}$ beam. Transmission from the ion source to the FMA entrance was generally around 30 percent. From this tune, we scaled the accelerator to the chosen m/q for the actinide beam.

The actinide production rates for this run were low. Rates varied based on laser performance and source conditions between 10 and 1000 actinide counts per second. Much of this low production can be attributed to the sample's low actinide concentration. Samples contained only 50-500ng of actinide in 5mgs of $\mathrm{Tb}_{2} \mathrm{O}_{3}$ matrix.. $\mathrm{Tb}_{2} \mathrm{O}_{3}$ was selected due to chemical similarities between the rare earth elements and actinides. However, in the course of our testing, we did not observe a benefit to the Tb matrix and the Tb produced long-term source contamination for other AMS experiments run using the ECR source. So, to avoid any mass conflicts with other AMS experiments, we will switch to an $\mathrm{Al}_{2} \mathrm{O}_{3}$ matrix. The actinide concentration in the sample will be increased to typically 1 percent, by atom, which will yield much higher actinide production form the source.

In terms of ion source efficiency, the ablated samples lost material at an average rate of $0.1 \mathrm{mg} / \mathrm{hr}$. Given a sample concentration of 500ng to $5 \mathrm{mg}$ of terbium, a total transport efficiency of 3 percent, including stripping efficiency at the FMA, and an average count rate of 1000ions/s 
at the FMA this gives an ion source efficiency of approximately $5 \times 10^{-6}$ into the selected charge state.

Following acceleration, the Fragment Mass Analyzer (FMA) was used for detection of the actinides. Figure 8 shows a schematic of the FMA. The FMA analyzes ions based on their m/q ratio, with a resolution of about 340, and like other AMS detection techniques, the detectors allow us to measure ion energy and specific energy loss, adding considerable discriminating power between the ion of interest and background ions [16]. Moreover, the FMA's physical dispersion of ions with different $\mathrm{m} / \mathrm{q}$ ratios along the focal plane allows us to block high-intensity background groups with a set of focal plane slits.

The base actinide, mass A (see equation (1) ), was measured first in the FMA, then the accelerator and the FMA were scaled to the $\mathrm{A}+1, \mathrm{~A}+2$, etc regions to look for major background contaminants in the unirradiated material. Because the ECR produces positive ions, many lower-mass species with the same $\mathrm{m} / \mathrm{q}$ will be accelerated with the actinide. To reduce the background counts in the detector, a $50 \mu \mathrm{g} / \mathrm{cm}^{2}$ carbon stripper foil is placed at the object of the FMA. The FMA is then scaled to one charge state lower than the accelerator so that the lower mass contaminants are separated on the focal plane. This stripping process reduces the total efficiency because most of the beam is spread into charge states which will not be detected in the FMA. Based on stripping calculations, approximately 15 percent of the initial beam is available in a single charge state. The ability to experimentally measure the charge state distribution is limited by the FMA's rigidity acceptances. However, because all the isotopes are detected at the FMA focal plane and all m/q's are accelerated to the same velocity, we can assume that the charge state distribution will be the same for $\mathrm{A}, \mathrm{A}+1$, etc and the stripping efficiency will cancel in the final ratio calculation. 
Figure 9 shows spectra for the $\mathrm{A}, \mathrm{A}+1$, and $\mathrm{A}+2$ regions for the Th sample. The accelerator is set to select $\mathrm{Th}^{37+}$ and the FMA is set to $\mathrm{Th}^{36+}$. Focal plane slits block the other charge states and most of the lower mass contaminants. The mass 232 region shows a Th peak clearly separated from the lower mass ${ }^{207} \mathrm{~Pb}$ contaminant. After scaling to mass 233 or 234 , the FMA will focus the respective mass in the same region of interest as established for the original 232 setting. These measurements show that there are, mostly, no major contaminants in the unirradiated sample. The measured sensitivities for this initial 2013 run were $5 \times 10^{-3}$ for the $A+1$ and $<1 \times 10^{-4}$ for the $A+2$. The lower limits on the expected ratios based on known cross sections and expected neutron fluence are $10^{-4}$ for $A+1$ and $10^{-8}$ for $A+2$. These sensitivities are primarily constrained by limited counting time, high rates from a source background due to a recent $\mathrm{Pb}$ experiment in the source, and low actinide production due to low concentration samples. Additional measurements performed in March 2015 with this same technique and higher concentration samples demonstrated significantly improved sensitivities down to $10^{-6}$ for A+1 and $10^{-8}$ for $A+2$. The results of this run are still under analysis and will be reported at a later date. However, given these preliminary results, we expect to be able to measure $\mathrm{A}+1$ for all samples and also measure $\mathrm{A}+2$ for most cases depending on the actinide cross talk.

For the ${ }^{237} \mathrm{~Np}$ sample we did see significant rates in these initial experiments, approximately 2 counts per second, for mass 238 from a combination of material in the sample and background material in the source. This provided us an opportunity to look at cross talk reduction with laser ablation. Figure 10 shows a series of successive measurements on the mass 238 region of the ${ }^{237} \mathrm{~Np}$ sample over the course of a few hours. The laser was turned on and off over the course of these runs and the count rates on the detector were measured. There is a baseline of 238 material from the source but the laser clearly introduces more 238 material from the sample. However, when the laser is turned off, the measured 238 count rate remains at the background level. This is 
an encouraging indication that the laser may help to reduce cross talk by limiting the buildup of material in the ion source.

\section{Conclusions}

We have developed a laser ablation material injection setup for the Electron Cyclotron Resonance Ion Source at ATLAS. Laser ablation offers finer control of the material injection rate, and a more favorable geometry to efficiently use material in the source compared to electrical sputtering. These benefits should help reduce cross talk between samples in the ion source, which is essential for the MANTRA project.

Through the use of feedback correction for the laser energy, and by rastering the laser across the sample, we have been able to produce low-intensity actinide beams with good stability. An initial experiment shows that ablation may help maintain source cleanliness. Additionally, recent improvements of the actinide yields compared with these initial experiments described here will allow us to measure the cross sections of interest.

More tests are currently underway to optimize the beam production. An experiment in March 2015 successfully measured the first irradiated samples at ATLAS. The results of this experiment are currently under analysis and will be reported separately.

\section{Acknowledgments}

This material is based upon work supported by the U.S. Department of Energy Office of Science, Office of Nuclear Physics, under contract number DE-AC02-06CH11357 and work 
authorization number KB/CH12/9/ARRA-3, FWP \#22695. This research used resources of ANL's ATLAS facility, which is a DOE Office of Science User Facility. 
References

[1] M. Salvatores, G. Palmiotti, G. Aliberti, H. Hiruta, R. McKnight, P. Obozinsky, and W.S. Yang, Nuclear Data Sheets, 109 (2008) 2725.

[2] G. Youinou, R. Vondrasek, H. Veselka, M. Salvatores, M. Paul, R. Pardo, G. Palmiotti, T. Palchan, O. Nusair, J. Nimmagadda, C. Nair, P. Murray, T. Maddock, S. Kondraskev, F.G. Kondev, W. Jones, G. Imel, C. Glass, J. Fonnesbeck, J. Berg, and W. Bauder, Nuclear Data Sheets, 119 (2014) 169.

[3] G. Youinou, G. Palmiotti, M. Salvatores, G. Imel, R. Pardo, F. Kondev and M. Paul, INL Internal Report, INL/EXT-10-17622 (2010) available at http://www.inl.gov/technicalpublications/Documents/4460753.pdf

[4] W. Kutschera, Int. J. Mass Spectrom., 242 (2005) 145.

[5] W. Kutschera, Int. J. Mass Spectrom., 349-350 (2013) 203.

[6] A.E. Litherland, Annu. Rev. Nucl. Part. Sci., 30 (1980) 437.

[7] M. Paul, D. Berkovits, I. Ahmed, F. Borasi, J. Caggiano, C.N. Davids, J.P. Greene, B. Harss, A. Heinz, D.J. Henderson, W. Henning, C.L. Jiang, R.C. Pardo, K.E. Rehm, R. Rejoub, D. Seweryniak, A. Sonzogni, J. Uusitalo, and R. Vondrasek, Nucl. Instrum. Methods Phys. Res., Sect B, 172 (2000) 688.

[8] M. Paul, R. Pardo, I. Ahmed, J. Greene, D. Henderson, R.V.F. Janssens, C.L. Jiang, K.E. Rehm, R. Scott, D. Seweryniak, and R. Vondrasek, Nucl. Instrum. Methods Phys. Res., Sect B, 294 (2013) 165.

[9] Alan L. Gray, Analyst, 110 (1985) 551.

[10] R. Harkewicz, J. Stacy, J. Greene, and R.C. Pardo, Rev. Sci. Instrum., 65 (1994) 1104.

[11] Richard Geller, Electron Cyclotron Resonance Ion Sources and ECRIS Plasmas, (Bristol, Philadelphia, 1998).

[12] T. Palchan, R. Pardo, F. Kondev, S. Kondrashev, C. Nair, R. Scott, R. Vondrasek, M. Paul, W. Bauder, P. Collon, G. Youinou, M. Salvatores, G. Palmiotti, J. Berg, T. Maddock, and G. Imel, ECRIS 2012 Conference Preceedings, www.JACoW.org, FRXA03 (2012) 190.

[13] E.G. Gamley, Physics Reports, 508 (2011) 91.

[14] Richard E. Russo, Xianglei Mao, Jhanis J. Gonzalez, and Jong Yoo, Spectroscopy, 28 (2013) 24.

[15] Oleg V. Borisov, Xianglei Mao, and Richard E. Russo, Spectrochim. Acta, Part B, 55 (2000) 1693. 
[16] Cary N. Davids and James D. Larson. Nucl. Instrum. Methods Phys. Res., Sect B, 40 (1989) 1224. 
Table I: Parameters of the laser used for ablation at the ECR.

\begin{tabular}{|l|l|}
\hline Laser Type & $\begin{array}{l}\text { Diode-Pumped } \\
\text { Nd:YAG }\end{array}$ \\
\hline Wavelength & $1064 \mathrm{~nm}$ \\
\hline Pulse Length & $15 \mathrm{ps}$ \\
\hline Energy per Pulse & $0-4 \mathrm{~mJ}$ Adjustable \\
\hline Repetition Rate & $0-400 \mathrm{~Hz}$ Adjustable \\
\hline Beam Profile & Gaussian \\
\hline $\begin{array}{l}\text { Beam Spot Size on } \\
\text { Sample }\end{array}$ & $0.5 \mathrm{~mm}$ \\
\hline
\end{tabular}


Figure 1: Floor plan of the ATLAS facility. The areas used for the MANTRA experiment are highlighted. The ECR ion source produces highly-charged positive ions which are then accelerated through just the first section of superconducting resonators to $1 \mathrm{MeV} / \mathrm{u}$. The Fragment Mass Analyzer (FMA) is used to separate ions based on their m/q ratio and they are detected with a position-sensitive gas detector and an ionization chamber to measure energy loss.

Figure 2: A cutaway of the ECRII ion source. A comparison of the geometry for electrical sputtering and laser ablation is shown.

Figure 3: A layout of the laser at the ECR. The laser is focused through the beam extraction line onto the sample in the back of the ECR source. The laser alignment is confirmed with a CCD camera. A backlight is used to locate the sample with the camera, shown in the top left corner.

Figure 4: Beam current of ${ }^{48} \mathrm{Ti}^{10+}$ compared with the energy output of the laser. The Ti beam current drops dramatically after 20 minutes due to the laser digging a crater in the sample.

Figure 5: A plot of the laser energy fluctuations with(a) and without(b) feedback correction.

Figure 6: Pictures of two samples post-ablation. The Ti sample on the left has been ablated without rastering the beam resulting in tight craters on the sample surface. The Tb sample on the right has been ablated while rastering the laser beam across the sample surface which eliminates the cratering.

Figure 7 A comparison of an ${ }^{27} \mathrm{Al}^{4+}$ beam with and without rastering. The beam current is much more stable while rastering the laser across the sample.

Figure 8: A schematic of the FMA. The design features two electric dipoles, ED1 and ED2, one magnetic dipole, MD1 and two quadrupole doublets, Q1 Q2 Q3 Q4. This QQEMEQQ configuration separates ions based purely on mass to charge ratio. The nominal mass resolution of the FMA is 340. The beam enters the FMA passing through a stripper foil to help separate low mass contaminants. At the focal plane, a Parallel Grid Avalanche Counter (PGAC) detects the position of the ions and an Ionization Chamber (IC) measures the energy loss 
Figure 9: Spectra of a Th sample first measuring the base actinide, ${ }^{232} \mathrm{Th}$, then scaling to the $\mathrm{A}+1$ and $\mathrm{A}+2$ regions. The lower mass contaminants are due to $\mathrm{m} / \mathrm{q}$ degeneracies from the ion source that cannot be separated by the accelerator. The double peaking seen in some of these spectra is a result of an overfocusing in the accelerator right before the FMA. This effect has been studied in a subsequent experiment and will be easily eliminated in future experiments.

Figure 10: Successive measurements of mass 238 in ${ }^{237} \mathrm{~Np}$ sample while turning the laser on and off. The accelerator is tuned for $\frac{238}{38+}$ and the FMA is set to $\frac{238}{37+}$. The introduction of ablated material does not appear to significantly raise the background rate of 238 . 
Fragment Mass Analyzer

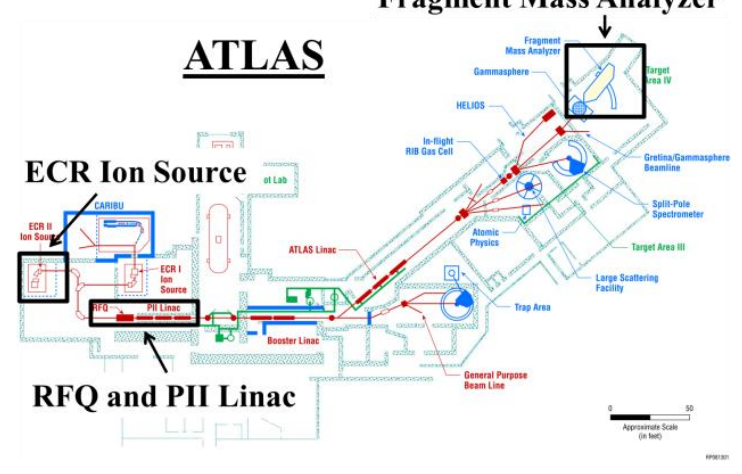

Figure 1

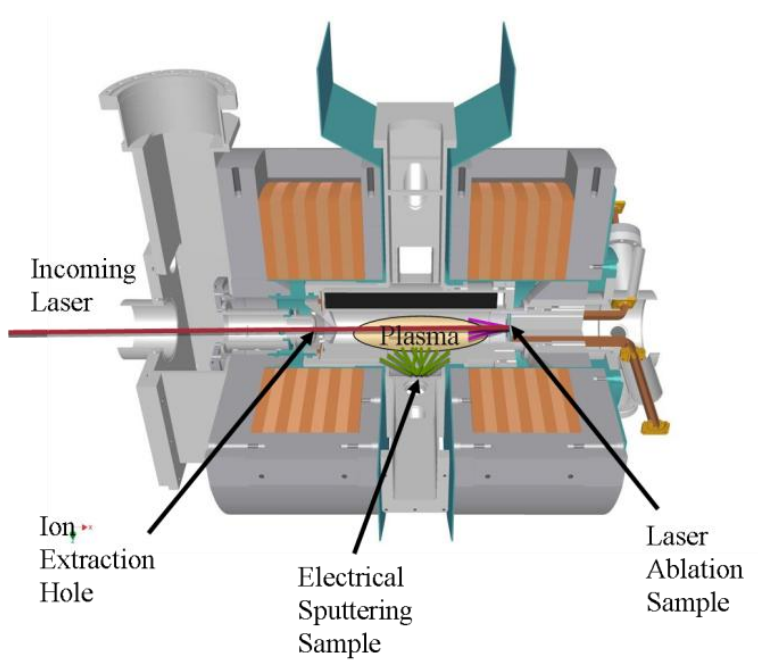


2

3

4

5

6

7

8

9

10

11

12

13

14

15

16

17

18

19

20

21

22

23

24

25

26

27

28

29

30

31

32

33

34

35

36

37

38

39

40

41

42

43

44

45

46

47

48

49

50

51

52

53

54

55

56

57

58

59

60

61

62

63

64

65

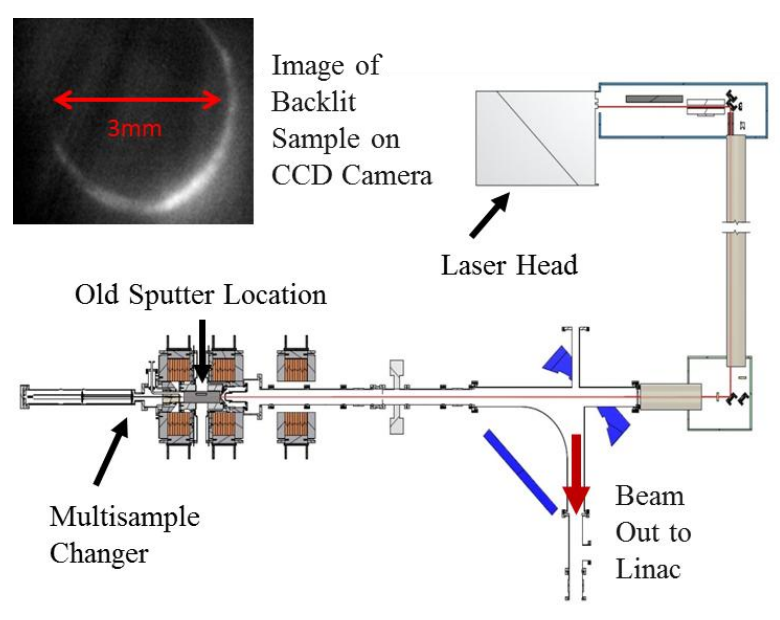

Figure 3

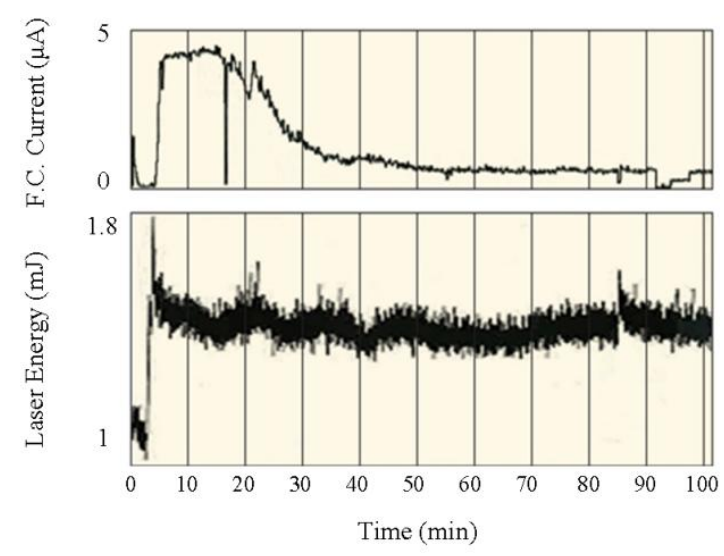

Figure 4 

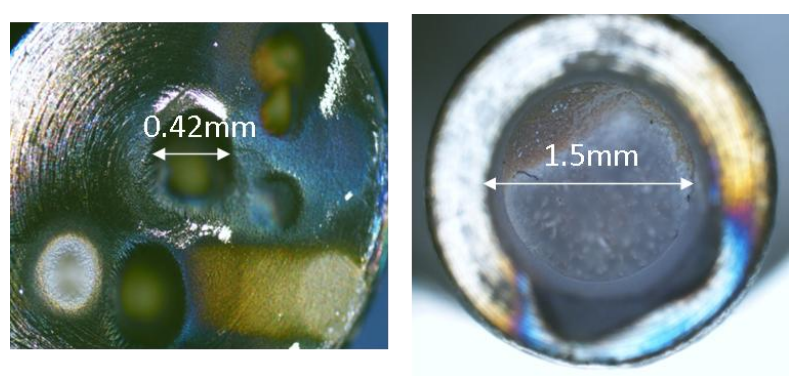

Figure 6

(b)

(a)

Figure 5 


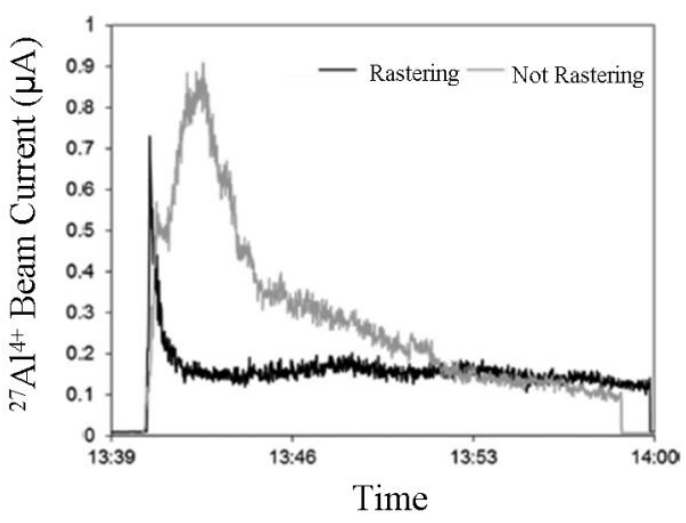

Figure 7

20

21

22

23

24

25

26

27

28

29

30

31

32

33

34

35

36

37

38

39

40

41

42

43

44

45

46

47

48

49

50

51

52

53

54

55

56

57

58

59

60

61

62

63

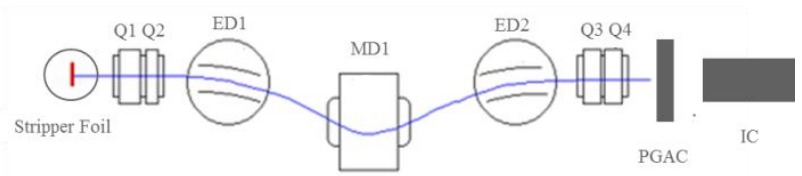

Figure 8 


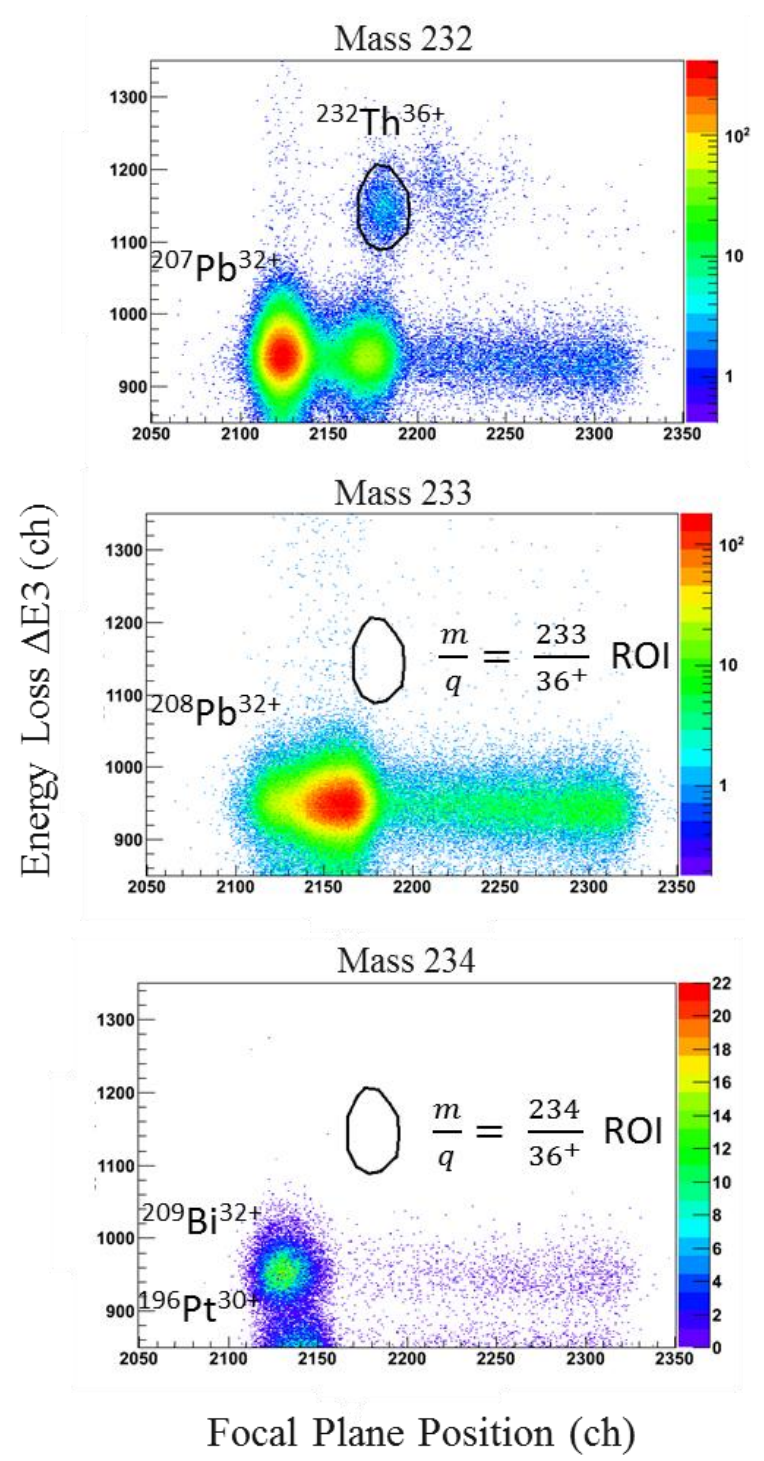

Figure 9 


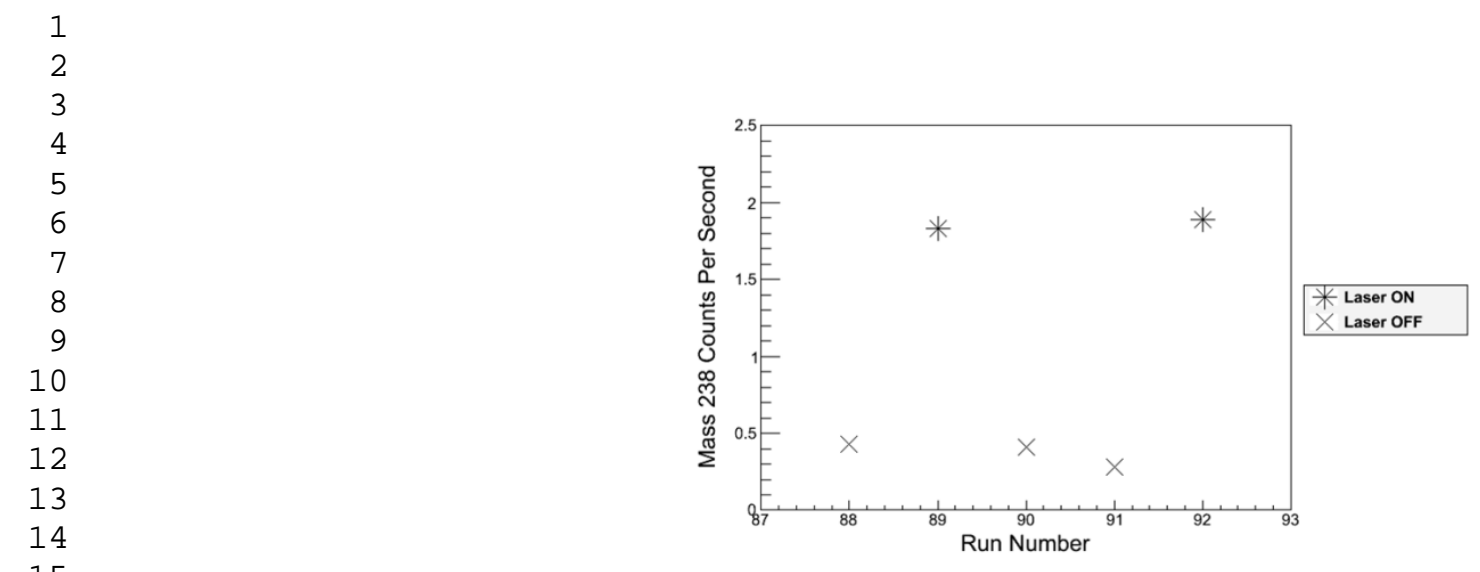

Figure 10 